\title{
HARDNESS AND MICROSTRUCTURE OF MIXED Al-CNF POWDER EXTRUSION
}

\begin{abstract}
In this study, mechanical properties and microstructures of extruded aluminum matrix composites were investigated. The composite materials were manufactured by two step methods: powder metallurgy (mixture of aluminum powder and carbon fiber using a turbular mixer, pressing of mixed aluminum powder and carbon fiber using a cold isostatic pressing) and hot extrusion of pressed aluminum powder and carbon fiber. For the mixing of Al powder and carbon fibers, aluminum powder was used as a powder with an average particle size of 30 micrometer and the addition of the carbon fibers was $50 \%$ of volume. In order to make mixing easier, it was mixed under an optimal condition of turbular mixer with a rotational speed of $60 \mathrm{rpm}$ and time of $1800 \mathrm{~s}$. The process of the hot-extrusion was heated at $450^{\circ} \mathrm{C}$ for 1 hour. Then, it was hot-extruded with a condition of extrusion ratio of 19 and ram speed of $2 \mathrm{~mm} / \mathrm{s}$. The microstructural analysis of extruded aluminum matrix composites bars and semi-solid casted alloys were carried out with the optical microscope, scanning electron microscope and X-ray diffraction. Its mechanical properties were evaluated by Vickers hardness and tensile test.
\end{abstract}

Keywords: Al-CNF composite, Cold Isotatic Presses, Powder extrusion, LED, microstructure

\section{Introduction}

Recently, there have been constant demands for Miniaturization and weight reduction, high-efficiency, environmentally friendly, multifunction in electronics industry such as mobile phone and automobile. Over past few years, several studies have been made on reduction energy consumption and environmental pollution. So LED lighting is integrated to the electronic device and increased energy efficiency [1-3]. However, if the electronic device is integrated, appliances generate high heat during operation. This may reduce the performance and lifetime of the products. For commercialization of electronic devices, it must be able to release heat effectively during device operation and the production cost should be low. Therefore, the concern with dissipating the heat generated by electronic material and possible operates for long periods of time has been growing. Study on the heat radiation material is being variously proceed such as polymers, ceramics, metal and etc. Among then, the most commonly used material is aluminum, because the price is cheaper than other materials [4-5]. Aluminum has excellent heat release properties; however, there is a need to enhance it for using materials in high-power electronics. There has been a renewal of interest in carbon - nano composite material that is made by added carbon in metal matrix metallic [6-7]. This study is basic research for developing the improved heat dissipation characteristics metallic carbon nano-composite. In this study, mechanical properties and microstructures of extruded Al-CNF composites were investigated.

\section{Experimental}

The material used in this study are pure aluminum powder (>99.xx) and the CNF type of pellet and it was mixed with each $50 \%$ volume percent. In this experiment, turbular mixer was used for producing a uniform mixed powder and charged stainless ball, pure aluminum powder in 2L HDPE container and mixed for 60 minutes at a speed of $60 \mathrm{rpm}$. In order to produce double Extrusion preform it was subjected to cold isostatic press at powder mixture. The conditions are gradually increased until a pressure $160 \mathrm{MPa}$ for 20 after initial hydrostatic. After reaching the target pressing force it was gradually reduced to maintain the pressure for 30 seconds. The fabricated molded body is then maintained for one hour at a temperature of 450 degree container using a 300 ton press, extrusion ratio 19:1 was hot-extruded under the conditions of Ram speed $2 \mathrm{~mm} / \mathrm{s}$ [8]. After then, it was an optical microscope, SEM, XRD, and the Vickers hardness test for the microstructure and mechanical property evaluation of the extruded material. 


\section{Results and discussion}

Fig. 1 shows the observed microstructure of the aluminum powder and an output type of the billet that are used in the study by SEM. Fig. 1a is an aluminum powder, average particle size $30 \mu \mathrm{m}$ in a relatively non-uniform shape. Fig. $1 \mathrm{~b}$ is the pellet-type CNF is made up of several hundred strands and the size of the average diameter is $8.7 \mu \mathrm{m}$. It was prepared extruding preform by cold isostatic press after uniformly mixing the powder in the turbular mixer.

Fig. 2 is an Al-C composite surface of a preform made by cold isostatic press. In aluminum matrix, itwas able to observe that the CNF, which are uniformly distributed. The format has the vertical direction, horizontal direction, a slanted form. $\mathrm{CNF}$ was made differently position when the preforms of the mixed powder before the cold isostatic press forming. It needs the additional study of the effect of CNF dispersion form and their properties during hot extrusion.

Fig. 3a shows a cross-section of the remaining butt after extrusion and metal flow pattern of the CNT-Al composite preform. Fig. $3 \mathrm{~b}$ is a surface of the extruded $\mathrm{Al}$ Composite CNF observed by stereo scopic microscope Fig. 3a shows the flow pattern observed in a typical extrusion direct extrusion of the material. It is a typical pattern in the friction at the container and die interfaces. In this case, it seems to form an extended dead zone and shear deformation band compared to the flow
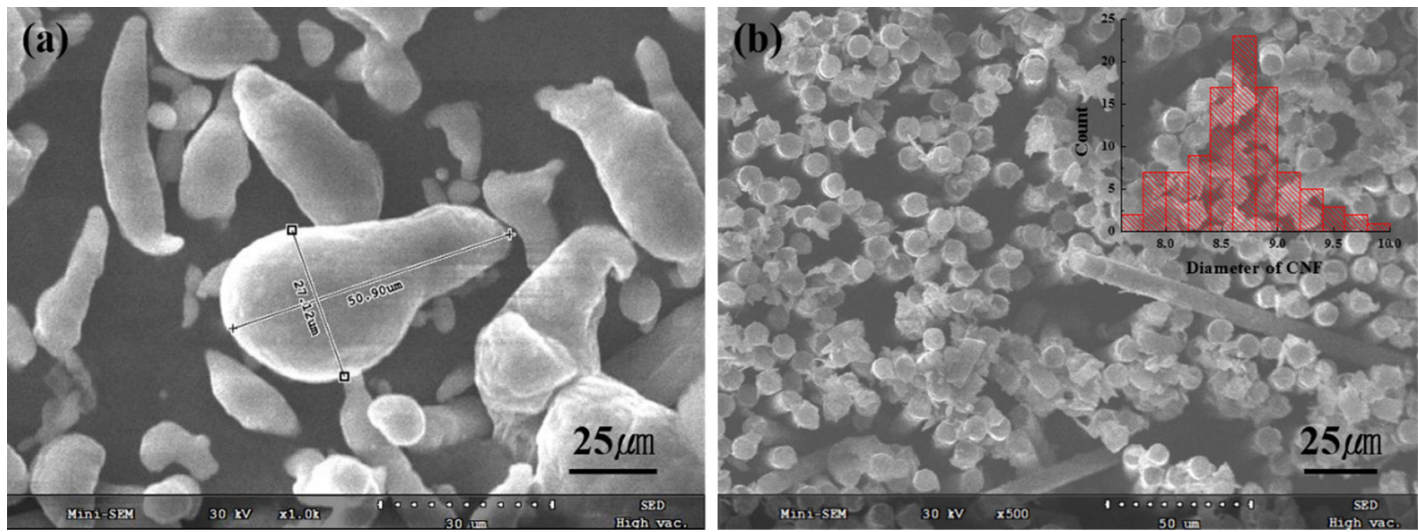

Fig. 1. SEM images of Al powder(a) and CNF pellet

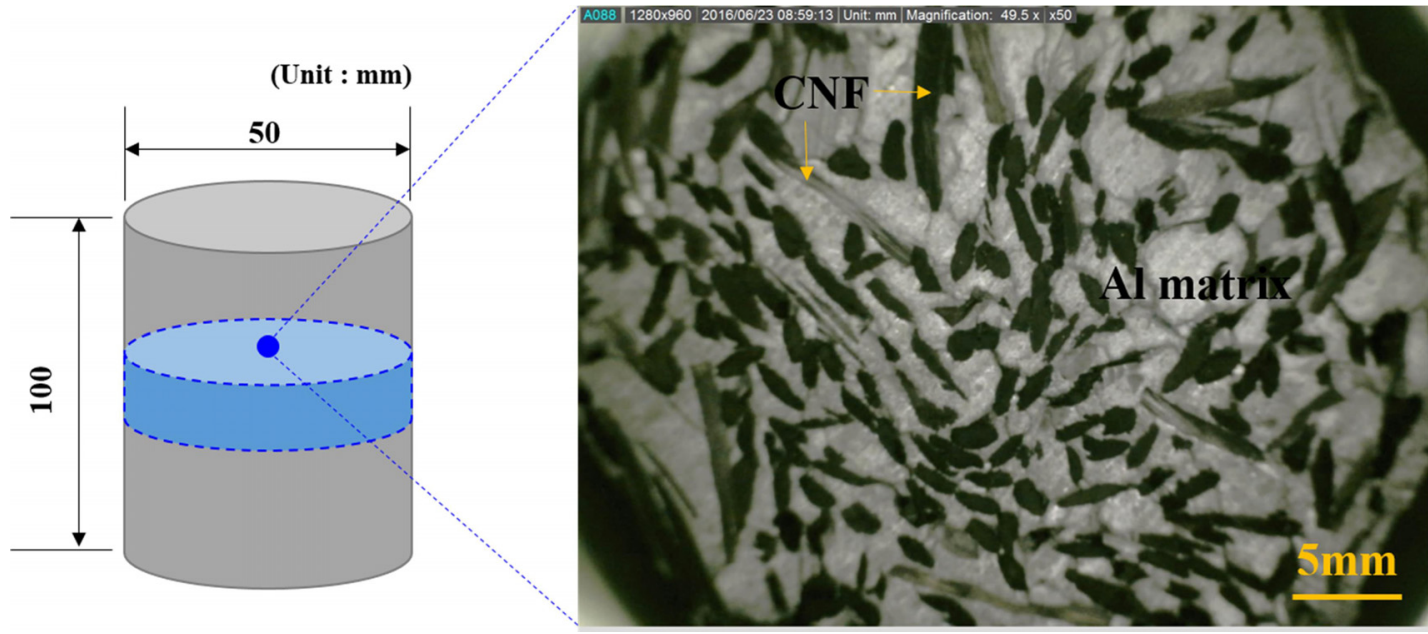

Fig. 2. Photomicrograph of Al-CNF composite preform fabricated by cold isostatic press
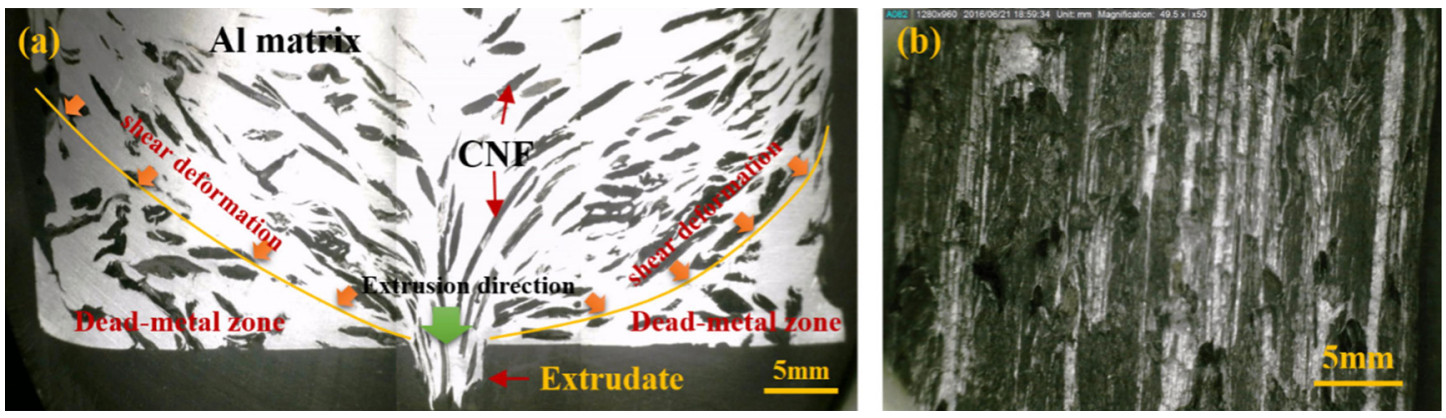

Fig. 3. Photomicrographs of (a) butt and (b) extruded Al-CNF composite after hot extrusion 
pattern of the indirect extrusion and non-uniform, depending on the direction of the extrusion material [9]. Fig. 3b is shown the surface of the Al-CNF composite has been stretched with each other shape to the extrusion direction and could find pick-up defects by the flow difference of $\mathrm{Al}$ and CNF during hot-extrusion. These defects appear in a shape that ends in the covering the start of the extrusion surface deposits. It needs optimum extrusion conditions for a sound extruded material manufactured: this is generated by the extrusion process parameters and the alloy homogenization conditions.
Fig. 4 shows a longitudinal section and cross section microstructures of the extruded Al-CNF composite. Most CNT pellet was aligned regularly in a compressed orientation within the aluminum matrix and aluminum powder and $\mathrm{CNF}$ pellet is extruded into each separate form without mixing. It could be observed some mixed form at the interface between the aluminum and fillet by extrusion.

Fig. 5 shows the surface of the extruded Al-CNF composites EDS mapping and diffraction results to analyze the generated phase and composition. As shown in Fig. 5a, oxide was distrib-
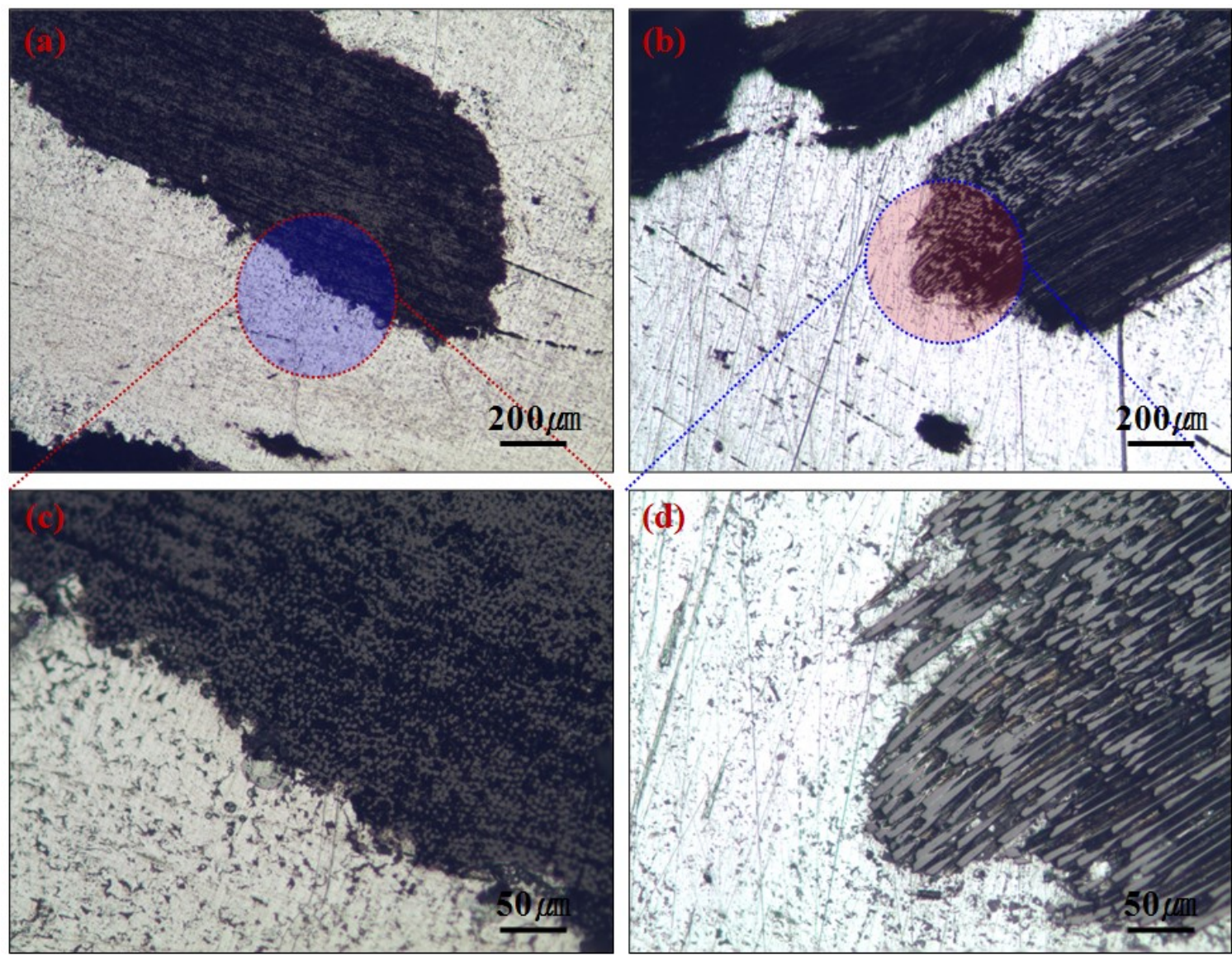

Fig. 4. Microstructures of extruded Al-CNF composites after hot extrusion;(a)(c) cross section and (b)(d) longitudinal section, (a)(b) $\times 50$ and (c)(d) $\times 200$
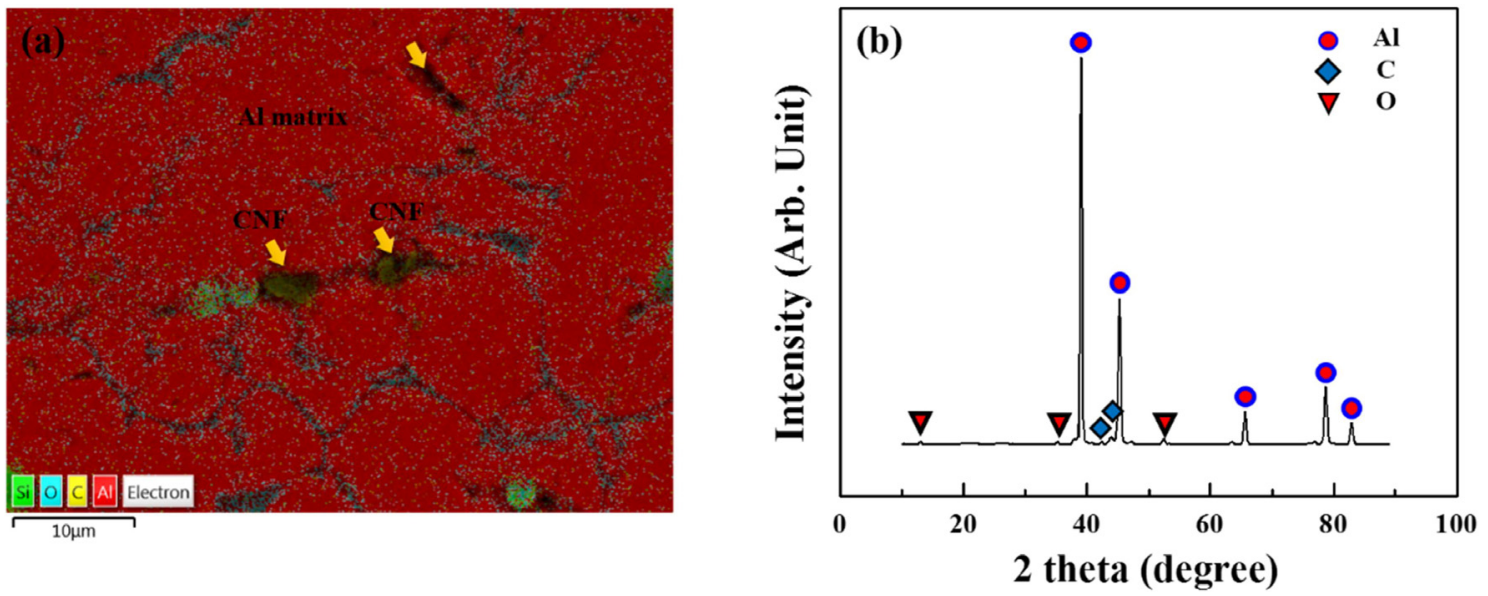

Fig. 5. EDS mapping result (a) and X-ray Diffraction(b) of extruded Al-CNF composites after hot extrusion 
uted in the aluminum matrix boundary and it could be observed in some of the interface between $\mathrm{Al}$ and CNF. In addition, it was found that expected distribution of $\mathrm{Si}$ in the grain boundary. This is presumably due to the influx part lubricant during the extrusion process. Fig. $5 \mathrm{~b}$ is XRD result and it could be obtained similar results in the EDS mapping. It was confirmed $\mathrm{Al}, \mathrm{C}$ and $\mathrm{O}$.

Fig. 6 displays the extrusion direction and the perpendicular direction of the micro-hardness of the extruded Al-CNF composites. In the case of pure aluminum, it has been reported with $30.3 \mathrm{Hv}$ micro hardness [12], however, in the case of Al-CNF composites increased with each $35.5 \mathrm{Hv}$ (Longitudinal section) and $35.9 \mathrm{Hv}$ (Cross section). This is determined as manufactured by extrusion Al-CNT composites interior grain size, the results were affected by the contribution of work hardening (strain hardening) and CNF. It is determined to have affected by the grain size, strain hardening of Al-CNF as manufactured by extrusion Al-CNT composites.

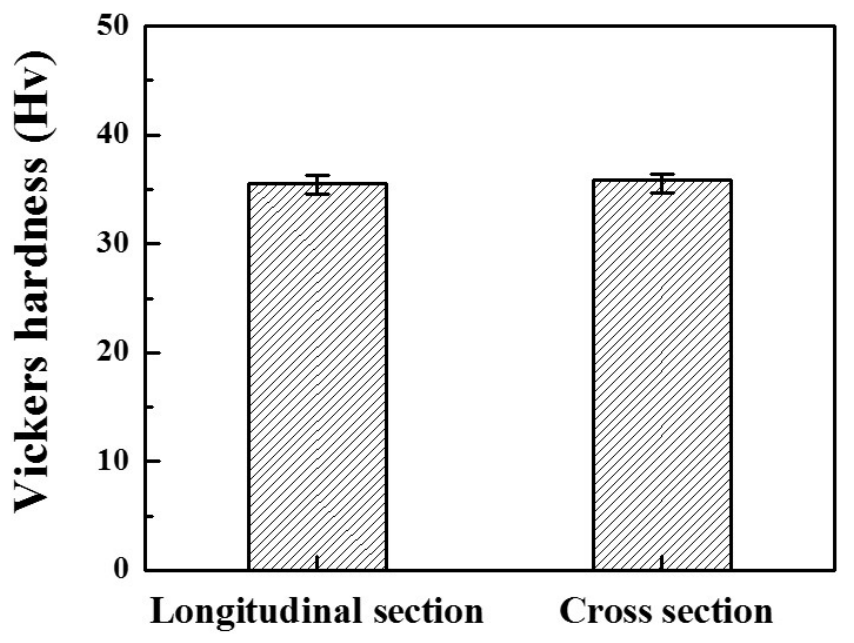

Fig. 6. Vickers hardness of extruded Al-CNF composite with different section.

\section{Conclusions}

In this study, it was mixed with $\mathrm{Al}$ powder and the pellet type of CNF in turbular mixer by using a cold isostatic press was prepared with Al-CNT composite preform by using a cold isostatic press was prepared with Al-CNT composite preform. After that, the hot extrusion to the Al-CNF preform was produced CNF-Al composites. It was performed to evaluate the microstructure and properties of the produced composite key results are as follows.

1) Al-CNF composites that is made by a cold isostatic press was suitable for manufacturing preforms for the oxidation, the preform and extruding the mixed powder compared to the canning process.

2) In the extrusion of Al-CNF composite preforms, it could be manufactured extrusion Al-CNF composite material, at the same time, when the CNT pellet dispersed in the direction of a non-uniform inside the preform to the extrusion direction is arranged a one-way. However, some defects were observed due to the difference in the extrusion $\mathrm{Al}$ and CNF's flow properties. Thus, the property evaluation is needed for optimum extrusion conditions and the application of heat sink material.

\section{Acknowledgments}

This work was supported by the Human Resource Training Program for Regional Innovation and Creativity through the Ministry of Education and National Research Foundation of Korea(NRF-2015H1C1A1035901)

\section{REFERENCES}

[1] J. Park, M.W. Shin, C.C. Lee, Optics Letters 29, 22 (2004).

[2] L. Yang, J. Hu and M.W. Shin, IEEE Electron Device Letters 29, 8 (2008).

[3] L. Kim, M.W. Shin, IEEE Trans. Component and Packaging Technology 30, 4 (2007)

[4] K. Shinozaki, A. Tsuge, Characterization Techniques of Ceramics: Development of high Thermal Conductive Aluminum nitride (in JPN), Ceramics, 21 (1986).

[5] H.L. Lee, S.M. Ha, Y. Yoo, S.-G. Lee, Polymer Science and Technology 24 (1), (2013).

[6] S.H. Yu, ICHMT 5 (13-14), 1609-1610 (2009).

[7] H.W. Kang, B.D. Kang, K.S. Park, Kase 51 (3), 12-19 (2008).

[8] P. Feltham, Metal Treatment 23, 440 (1956) CA 94305, June.

[9] P.K. Saha, ASM international, Aluminum Extrusion Technology, pp.1-10 (2000.)

[10] L.J. Matienzo, K.J. Holub, W. Vandatta, Applications of Surface Science 15, 307-320 (1982).

[11] T. Minoda, H. Hayakawa, S. Matsuda, H. Yoshida, $7^{\text {th }}$ international aluminum extrusion technology seminar, 23-29 (2000).

[12] K. Kang, H. Park, C. Lee, J. Kor. Inst. Surf. Eng. 45 (1), 1-7 (2012). 